\title{
edoc
}

Institutional Repository of the University of Basel

University Library

Schoenbeinstrasse 18-20

CH-4056 Basel, Switzerland

http://edoc.unibas.ch/

Year: 2014

\section{Der Europäische Gesetzgeber im Lichte des neu gefassten § 136 Abs. 1 Satz 2 StPO - Gespenst, Phantom oder guter Geist?}

Gless, Sabine

Posted at edoc, University of Basel

Official URL: http://edoc.unibas.ch/dok/A6328837

Originally published as:

Gless, Sabine. (2014) Der Europäische Gesetzgeber im Lichte des neu gefassten § 136 Abs. 1 Satz 2 StPO Gespenst, Phantom oder guter Geist? In: Streitbare Strafrechtswissenschaft. Berlin, S. 1059-1072.

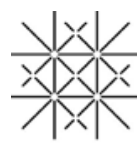




\title{
Der Europäische Gesetzgeber im Lichte des neu gefassten $\$ 136$ Abs. 1 Satz 2 StPO - Gespenst, Phantom oder guter Geist?
}

\author{
SABine GLESS
}

\section{Die Brüssler Strafrechtspflege vor den Toren}

„Ein Gespenst gebt um in Europa“, warnte Bernd Schünemann im Jahr 2002 die deutsche Strafrechtswissenschaft und meinte die „Brüsseler ,Strafrechtspflege“", 1 die er auf das nationale Strafrecht zukommen sah. Tatsächlich haben EU-Initiativen wie der Europäische Haftbefehl oder die Fahndung über das Schengener Informationssystem (SIS) die Praxis der Strafverfolgung schnell verändert - jedoch zunächst in grenzüberschreitenden Fällen. ${ }^{2}$ Bis der EU-Gesetzgeber im Kern der deutschen Strafprozessordnung auf der Arbeitsebene wahrnehmbare Veränderungen bewirkt hat, verging ein weiteres Jahrzehnt, und es geschah überraschenderweise nicht klar und einseitig als Abbau rechtsstaatlicher Grundsätze zum Nachteil von Beschuldigten. ${ }^{3}$

Anstoß für eine Reform im Kerngebiet gab vielmehr eine Initiative, mit welcher der EU-Gesetzgeber die Beschuldigtenstellung europaweit festigen will: Die EU-Richtlinie 2012/13 über das Recht auf Belehrung und Unterrichtung in Strafverfahren ${ }^{4}$ (im Folgenden: EU-Richtlinie 2012/13) veranlasste den deutschen Gesetzgeber die Informations- und Belehrungsrechte und das Recht auf Übersetzung ${ }^{5}$ auszubauen. Das Gesetz zur Stärkung der Verfahrensrechte von Beschuldigten im Strafverfahren ergänzte unter anderem $\mathbb{1 3 6}$ Abs. 1 Satz 2 StPO: ${ }^{6}$ Heute wird die beschuldigte Person in Deutschland nicht nur darauf hingewiesen, dass es ihr nach dem Gesetz freisteht, sich zu der Beschuldigung zu äussern oder nicht zur Sache auszusagen,

1 Schünemann GA 2002, $501 \mathrm{ff}$.

2 Satzger Internationales und Europäisches Strafrecht, 6. Aufl. 2013, \10 Rn. 1 ff.

3 Schünemann GA 2002, 501, 513.

${ }^{4}$ Richtlinie 2012/13/EU des Europäischen Parlaments und des Rates vom 22. Mai 2012 über das Recht auf Belehrung und Unterrichtung in Strafverfahren, ABl. L 142 vom 1.6.2012, 1 .

${ }^{5}$ Siehe dazu die Neufassung von $₫ 187$ Abs. 1 GVG.

${ }^{6}$ Zum Gesetzgebungsverfahren BT-Drucks. 17 / 12578 und 17 / 13528. 
und dass sie jederzeit einen von ihr zu wählenden Verteidiger befragen darf, sie „ist ferner darüber zu belehren, daß sie zu ibrer Entlastung einzelne Beweiserbebungen beantragen und unter den Voraussetzungen des $\int 140 \mathrm{Ab}$ satz 1 und 2 die Bestellung eines Verteidigers nach Maßgabe des $\int 141 \mathrm{Ab}$ satz 1 und 3 beanspruchen kann."

Ist die Brüssler Strafrechtspflege also nicht, oder jedenfall nicht immer, das Schreckgespenst, das man zu Beginn dieses Jahrtausends heraufziehen sah? Ist es vielleicht nur ein Phantom, das zwar - wie der Jubilar an vielen Stellen rhetorisch brillant herausgearbeitet hat - die Prämissen unserer Kriminalpolitik verändert hat, ${ }^{7}$ am Ende aber doch nicht so schlimm und manchmal sogar ein guter Geist ist, dessen Hand und Schrift es sorgfältig zu beobachten gilt und mit dem es sich zu reden lohnt? Mit Blick auf die jüngste Reform des $\$ 136$ StPO dürfte ein unbefangener Beobachter durchaus Letzteres konzedieren. Denn die auf den Weg gebrachten EU-Vorgaben an die Mitgliedstaaten scheinen den Warnungen Rechnung zu tragen, die in Deutschland immer wieder unter anderem engagiert von Bernd Schünemann vorgetragen wurden: Auf Weisung aus Brüssel müssen in der EU mit strafrechtlichen Vorwürfen konfrontierte Personen nach europäisch einheitlich definierten Vorgaben belehrt und unterrichtet werden. ${ }^{8}$ Damit ist anscheinend auch eine der Kernforderungen erfüllt, die Bernd Schünemann im Rahmen des von ihm initiierten Projekts für „Ein Gesamtkonzept für die europäische Strafrechtspflege" erhoben hat."

Eine Gabe ist jedoch nicht immer eine reine Wohltat. Das gilt bei europäischen Vorgaben schon deshalb, würde der Jubilar zu bedenken geben, weil jede europaweite Festlegung grundsätzlich eine Harmonisierung bedeute, welche - wie er ebenfalls in verschiedenen Werken herausgearbeitet hat - an sich die Gefahr des "race to the bottom" mit sich bringe. ${ }^{10}$ Dass der EUGesetzgeber dieser Gefahr vorzubeugen sucht, indem er es den Mitgliedstaaten, wie auch in der EU-Richtlinie 2012/13, freistellt, auf nationaler Ebene weiter gehende Individualrechte beizubehalten, ist an sich begrüßenswert, aber zugegebenermaßen in der Praxis weder eine echte Prophylaxe noch gänzlich unproblematisch. ${ }^{11}$

7 Schünemann StV 2003, 531, 533 warnte den deutschen Gesetzgeber zum „Lakai von Brüssel zu schrumpfen“ und forderte nationalstaatliche Umsetzungsspielräume, vgl. a. Schünemann/Roger ZIS 2010, 515, $516 \mathrm{ff}$.

${ }^{8}$ Dazu KOM (2010) 392 endg. vom 20.7.2010.

9 Vgl. Vorschläge Schünemann in: ders. (Hrsg.), Gesamtkonzept für die europäische Strafrechtspflege - A Programme for European Criminal Justice, 2006.

${ }^{10}$ Dazu mit Blick auf die Verteidigung: Nestler in: Schünemann (Hrsg.), Gesamtkonzept für die europäische Strafrechtspflege - A Programme for European Criminal Justice, 2006, S. $166,168$.

${ }^{11}$ Vgl. Erwägungsgrund 40 sowie Art. 10, RL 2012/13/EU. 


\section{II. $\ 136 \mathrm{StPO}$ - und der europäische Geist}

Bernd Schünemann hat das Gespenst einer das nationale Recht prägenden EU-Strafrechtsgesetzgebung vor gut einem Jahrzehnt zu Recht an die Wand gemalt: Die Zusammenarbeit der EU-Staaten zielte zunächst praktisch ausschließlich auf die Vereinfachung der zwischenstaatlichen Kooperation zur Strafverfolgung. Welchen Einfluss dies auf Beschuldigten- oder Bürgerrechte haben würde, interessierte kaum jemanden. Das Zusammenwirken der Behörden drohte, die in den nationalen Rechtsordnungen über lange Zeit gewachsene Balance zwischen staatlicher Strafverfolgungsmacht, Beschuldigteninteressen, Bürgerrechten und Opferanprüchen ins Ungleichgewicht zu bringen. Eine Lösung schien nicht in Sicht, schon weil der primär durch Wirtschaftsinteressen und eine Logik der ökonomischen Integration getriebene europäische Zentralisierungsprozess gar nicht das Instrumentarium vorsah, um auf Besonderheiten der Strafverfolgung Rücksicht zu nehmen. Hinzu kommt, dass Deutschland einen Standard im Strafverfahren erreicht hat, der von den inländischen Akteuren des Strafverfahrens als positiv und jedenfalls einem europäischen Anspruch auf (Neu-)Regelung gegenüber - als erhaltungswürdig angesehen wird. ${ }^{12}$

\section{Ausgangslage im deutschen Recht}

Den europäischen Bestrebungen stand man demgegenüber desinteressiert bis ablehnend gegenüber, denn der europäische Gesetzgeber drohte den deutschen Standard zu stören. Das gilt auch für die im deutschen Strafverfahren im Vergleich zu manchen anderen EU-Staaten weit gehenden Belehrungspflichten. Man ist in Deutschland um eine Belehrung in "korrekter" Weise bemüht, ${ }^{13}$ und selbst wenn ein Beschuldigter bereits einen Wahl- oder Pflichtverteidiger hat, besteht noch eine Hinweispflicht. ${ }^{14}$ Denn es könnte beispielsweise sein, dass er mit diesem Verteidiger noch nicht oder noch nicht eingehend gesprochen hat und nicht weiß, dass er sich vor der Vernehmung mit ihm beraten kann. ${ }^{15}$ Die Rechtsprechung hat darüber hinaus weitere

${ }_{12}$ Zur Sicht des nationalen Gesetzgebers vgl. BR-Drucks. 155 / 03 vom 23.5.2003; zur Sicht der Praktiker, die einen „Einheitsbrei aus Brüssel“ fürchten, vgl. die Auswertung von Praktikerinterviews durch Wabl in: Vernimmen-Van Tiggelen/Surano/Weyembergh (Eds.), The future of mutual recognition in criminal matters in the European Union, Brüssel 2009, S. 115 ff. sowie zur Komplexität dieses Unterfangens etwa: Vernimmen-Van Tiggelen/ Surano in: Vernimmen-Van Tiggelen/Surano/Weyembergh a.a.O., S. $570 \mathrm{ff}$.

${ }_{13}$ Dazu Hamm NJW 1996, 2185 ff.; Löwe/Rosenberg/Gleß StPO, 26. Aufl. 2007, $\$ 136$ Rn. 40.

${ }^{14}$ Strittig, vgl. dazu: B. Meble NJW 2007, 969, 973.

15 Roxin JZ 1993, 426; vgl. a. BGHSt 38, 375. 
Pflichten etabliert, die den Zugang zum Verteidiger faktisch sichern sollen. ${ }^{16}$ Bereits vor der Ergänzung von $\$ 136$ Abs. 1 Satz 2 StPO war anerkannt, dass ein mittelloser Beschuldigter, der einen Verteidiger wünscht, auf die Möglichkeit der Pflichtverteidigung und Beratung durch den anwaltlichen Notdienst aufmerksam gemacht werden muss, ${ }^{17}$ wobei die Einzelheiten streitig blieben. Vor diesem Hintergrund ist verständlich, dass der deutsche Gesetzgeber selbst nur geringen Änderungsbedarf infolge des Inkrafttretens der EU-Richtlinie sah. Geboten war die ausdrückliche Verankerung der Belehrungspflicht über die Möglichkeit unentgeltlicher Verteidigung. Denn die Frage, ob bzw. unter welchen Voraussetzungen der Beschuldigte über die Möglichkeit kostenloser Strafverteidigung eigens zu belehren ist, war in Deutschland, entgegen Aussagen in den Gesetzesmaterialien, nicht abschließend geklärt. ${ }^{18}$

\section{Rechtslage nach der Reform}

Den Streit über die Ausgestaltung dieser Belehrungspflicht beendet die Gesetzesänderung mit der Reform des $\ 136$ StPO. Nunmehr ist über das Recht zur unentgeltlichen Beiordnung eines Pflichtverteidigers bei richterlichen und - nach $\ 163$ a Abs. 3 S. 2 und Abs. 4 S. 2 - auch bei staatsanwaltschaftlichen und polizeilichen Vernehmungen zu belehren. Dies betrifft Fälle der notwendigen Verteidigung sowohl in den enumerierten Fällen nach $₫ 140$ Abs. 1 als auch nach den allgemeinen Voraussetzungen in $\mathbb{1} 140$ Abs. 2 StPO. ${ }^{19}$ Der Verweis auf $\mathbb{} 141$ Abs. 1 und 3 StPO stellt klar, dass der Beschuldigte insbesondere darüber zu belehren ist, dass ihm bereits im Ermittlungsverfahren ein Verteidiger zu bestellen ist, wenn die Staatsanwaltschaft dies wegen des erkennbaren Bestehens notwendiger Verteidigung beantragt. ${ }^{20}$ Damit sind Beschuldigte - jedenfalls auf dem Papier - durch die Reform heute im Strafverfahren besser gestellt als im Vergleich zu der Situation vor Umsetzung des EU-Rechts.

16 BGHSt 38, 214; 38, 372 = JR 1993, 332 mit Anm. Rieß = JZ 1993, 425 mit Anm. Roxin; BGHSt 42, 15, 26 = StV 1996, 187 m. Bespr. Müller; BGHSt 42, 170; 47, 233; BGH NStZ 2006, $236=\mathrm{StV}$ 2006, 567 mit Anm. Beulke/Barisch.

17 BGHSt 47, 233; BGH NStZ 2006, 236.

18 Vgl. Meyer-Goßner StPO, 56. Aufl. 2013, \136 Rn. 10; LR/Lüderssen/Jahn, 26. Aufl. 2007, 137 Rn. 66b; Woblers FS Rudolphi, 2004, S. 713, 731; B. Meble, Zeitpunkt und Umfang notwendiger Verteidigung im Ermittlungsverfahren, 2005, S. 305.

${ }_{19}$ Gesetzentwurf der Bundesregierung, Entwurf eines Gesetzes zur Stärkung der Verfahrensrechte von Beschuldigten im Strafverfahren, BT-Drucks. 17 / 12578, Begründung zu Nummer 3.

${ }^{20}$ Beschlussempfehlung und Bericht des Rechtsausschusses zu dem Gesetzentwurf der Bundesregierung, Entwurf eines Gesetzes zur Stärkung der Verfahrensrechte von Beschuldigten im Strafverfahren (Drs. 17/12578), BT-Drucks. 17 / 13528, S. 4. 
Es fehlen jedoch flankierende Regelungen. So ist etwa auch in Deutschland umstritten, welche Konsequenzen aus einer fehlenden Belehrung über das Recht auf Pflichtverteidigung folgen. Der $\mathrm{BGH}^{21}$ und ein Teil der Literatur ${ }^{22}$ haben ein Beweisverwertungsverbot bei Verletzung einer - sich dann nur im Einzelfall ergebenden - Belehrungspflicht abgelehnt. Diese Ansicht muss angesicht der Reform überdacht werden. Die europäisch initiierte Neuregelung stärkt diejenigen, die ein Beweisverwertungsverbot befürworten, wenn Beschuldigte über ihre Möglichkeiten einer unentgeltlichen Verteidigerbestellung nicht belehrt worden sind. ${ }^{23}$ Nach der Ergänzung von $\ 136$ Abs. 1 S. 2 StPO ist der Fall, dass der Beschuldigte nicht über das Recht auf unentgeltliche Verteidigung belehrt wurde, ebenso wie die Verletzung anderer Vorschriften zu behandeln, die den ungehinderten Zugang zu einem Verteidiger sichern sollen. ${ }^{24}$ Wenn man das Recht auf Verteidigerkonsultation auch für mittellose Beschuldigte ernst nimmt, ${ }^{25}$ muss eine Verletzung der Belehrungspflicht grundsätzlich zu einem Verwertungsverbot führen. ${ }^{26}$

\section{Abbau rechtsstaatlicher Verfahrensgarantien - nur ein Phantom?}

Die Ambition der EU-Richtlinie 2012/13 und deren Konsequenzen für das deutsche Recht führt damit zur Frage, ob der europäische Gesetzgeber in seiner wahren Gestalt ursprünglich verkannt wurde oder ob sich in seiner Erscheinungsform verändert hat, da er nun Individualrechte, jedenfalls das Recht auf Belehrung und Unterrichtung in Strafverfahren, schützt und fördert. Hat sich als Phantom entpuppt, was als Gefahr interpretiert wurde, und könnte es sich im Laufe der Zeit gar zu einem guten Geist entwickeln?

\section{EuGH als Hüter der Individualrechte}

Tatsächlich existierte auf europäischer Ebene von Anfang an ein guter Geist für die Bewahrung und den Ausbau von Individualrechten, allerdings versteckt in der EU-Logik, gegründet auf die EU-Freiheiten, die in jedem

21 BGH NStZ 2006, 236, 237.

22 B. Meble (Fn. 18), S. 343.

23 Klemke StV 2003, 413, 415; Schlothaner/Weider StV 2004, 504, 515.

${ }^{24}$ LR/Gleß (Fn. 13), $\$ 136$ Rn. 95 ff. sowie zur qualifizierten Belehrungspflicht: Neuhaus $\mathrm{StV} 2010,45,50 \mathrm{ff}$.

${ }^{25}$ BGHSt 38, 214; 38, 372 = JR 1993, 332 mit Anm. Rieß = JZ 1993 425, mit Anm. Roxin; BGHSt 42, 15 = BGH StV 1996 187, 189, mit Anm. Egon Müller ebenda S. 353 = NStZ 1996, 291, mit Anm. Beulke ebenda S. 257; Rieß JR 1993, 334.

${ }^{26}$ Vgl. BVerfGE 46, 210; BGHSt 38, 214; 38, 372; 39, 352; 47, 173; Eisenberg Beweisrecht der StPO, 8. Aufl. 2012, Rn. 373 ff. 
Rechtsgebiet, also auch im Strafrecht zugunsten einzelner Betroffener wirken. So hat sich der Europäische Gerichtshof (EuGH) in Luxemburg in dreierlei Hinsicht als Hüter der in den Verträgen verbrieften Individualrechte hervorgetan: ${ }^{27}$ Seit Beginn der europäischen Integration hat der EuGH nach der Prämisse entschieden, dass wer rechtmäßig eine europäische Grundfreiheit in Anspruch nimmt, straflos bleiben muss, auch wenn er nationales Strafrecht verletzt. ${ }^{28}$ Denn das mitgliedstaatliche Recht ist dem EG-Recht insoweit untergeordnet. ${ }^{29}$ In gleicher Weise hat der EuGH in seiner frühen Funktion als Schiedsgericht die ersten Gehversuche der gemeinsamen $\mathrm{Zu}$ sammenarbeit zur Strafverfolgung mit einer Blaupause einer Idee eines gemeinsamen europäischen Strafrechtsraumes durch die Rechtsprechung ${ }^{30}$ zur europaweiten Geltung des Strafklageverbrauches nach Art. 54 f. Schengener Durchführungsübereinkommen (SDÜ) ${ }^{31}$ unternommen: Es hob die Urteile der mitgliedstaatlichen Gerichte auf, die das Verbot der Doppelbestrafung auf eine gerichtliche Verurteilung beschränkten und entschied, dass jede rechtskräftige Entscheidung einer zuständigen Instanz in einem EU-Staat einen schengenweiten Strafklageverbrauch auslöst, sofern sie auf einer Tatsachen- und Rechtsprüfung beruht..$^{32}$ Deshalb verbrauchen nicht nur Urteile in einem anderen Schengen-Staat die Strafklage, ${ }^{33}$ sondern auch verfahrensbeendende Absprachen. ${ }^{34}$ Die Luxemburger Richter bejahen ein grenzüberschreitendes Verfolgungsverbot ferner, wenn die Tat in einem anderen Schengen-Staat aus anderen Gründen unverfolgbar ist, etwa aufgrund von Verjährung. ${ }^{35}$ Der EuGH ist den Bedürfnissen der Strafverteidigung auch

${ }^{27}$ Grundsätzlich zum Vorrang von EG-Recht: EuGH, Urt. v. 15.7.1964, Costa ./. E.N.E.L., Rs. C-6/64.

${ }_{28}$ Satzger (Fn. 2), $\$ 9$ Rn. 81; Hecker Europäisches Strafrecht, 4. Aufl. 2012, $\$ 9$ Rn. 24 f., $33 \mathrm{ff} ., 36 \mathrm{ff}$.

${ }^{29}$ EuGH, Urt. v. 7.2.1979, Ministère public ./. Auer, Rs. C-136/78, Slg. 1979, 437, 448 ff., Rn. $19 \mathrm{ff}$.

${ }^{30}$ EuGH, Urt. v. 18.7.2007, Kretzinger, Rs. C-288/05; vgl. im Einzelnen: Lagodny NStZ 1997, 265; Schomburg NJW 1999, 540; Bohnert/Lagodny NStZ 2000, 636; Radtke/Busch NStZ 2003, 281; Stein NJW 2003, 1162 ; Vogel/Norouzi JuS 2003,1059; Woblers FS Eisenberg, 2009, S. 807, $816 \mathrm{ff}$.

31 Übereinkommen zur Durchführung des Übereinkommens von Schengen vom 19.6.1990, BGBl. II 1993, S. 1013; BGBl.1904, II 1994, S. 631; vgl. dazu Hecker (Fn. 28), \13 Rn. $12 \mathrm{ff}$.

32 Vgl. EuGH, Urt. v. 11.2.2003, Strafverfahren gegen Hüseyin Gözütok und Klaus Brügge, Rs. C-187/01, C-385/01; Radtke/Busch NStZ 2003, 281, 284; Stein NJW 2003, 1162.

${ }_{33}$ Vgl. EuGH, Urt. v. 28.9.2006, van Straaten ./. Niederlande und Italien, Rs. C-150/05, StV 2007,57, Rn. 54 ff.

${ }^{34}$ EuGH, Urt. v. 10.3.2005, Strafverfahren gegen Filomeno Mario Miraglia, Rs. C-469/03, NJW 2005, 1337.

35 EuGH, Urt. v. 28.9.2006, Strafverfahren gegen Giuseppe Francesco Gasparini u.a., Rs. C-467/04, StV 2007, 113; Urt. v. 11.12.2008, Klaus Bourquain, Rs. C-297/07, NStZ 2009, 454. 
prozessual entgegen gekommen und hat für die Vorabentscheidungsverfahren in Strafsachen ein Eilverfahren geschaffen und so das Problem der langen Verfahrensdauer entschärft. ${ }^{36}$

Darüber hinaus hat der EuGH mit seiner Rechtsprechung zu den allgemeinen Rechtsgrundsätzen Eingriffe in Rechte der Bürger, insbesondere Akte mit repressiver Wirkung für den Einzelnen, in eine justizförmige Struktur überführt. Mit Hilfe der aus übergeordneten Rechtsgrundsätzen abgeleiteten Prinzipien garantieren die Luxemburger Richter ein rechtstaatliches Verfahren, Rechtsschutz und ein Minimum an Verteidigungsrechten. ${ }^{37}$ Die Rechtsprechung prägt die Kartellstrafverfahren und Disziplinarverfahren auf EU-Ebene ebenso wie die vor-Ort-Kontrollen durch das Europäische Amt zur Betrugsbekämpfung. ${ }^{38}$ Des Weiteren haben sie zu einer Restrukturierung der "targeted sanctions“ geführt. $^{39}$

\section{EU-Kommission als Initiatorin für neues Rechtsdenken}

Die EU-Kommission bemüht sich ebenfalls, den neu zu gestaltenden Raum der Freiheit, der Sicherheit und des Rechts nicht nur als Strafverfolgungsraum auszubauen, sondern Individualrechte zu schützen. ${ }^{40}$ Dafür nutzt sie die Folgenabschätzung im Grundrechtsbereich, also die Aufforderung an die gesetzgebenden Organe bei jeder neuen EU-Maßnahme die Konsequenzen für die Grundrechte- auch die Justizgrundrechte des Einzelnen zu bedenken, das Vorstufe eines "mainstreamings“ zugunsten der Verfahrensrechte sein könnte. ${ }^{41}$ Die EU-Organe sind zu solchen Überlegungen bereits seit 2001 angehalten. ${ }^{42} 2005$ forderte eine Mitteilung der Kommission die Berücksichtigung der EU-Grundrechtecharta in Rechtsetzungsvorschlägen im Sinne eines bestimmten methodisches Vorgehens, ${ }^{43} 2010$ schließlich betonte die Justizkommissarin, dass es einer Grundrechtsfolgeabschätzung

${ }^{36}$ Dazu: ABl. C 64 vom 8.3.2008, 1.

37 Das wird etwa deutlich an dem pointierten Schlussantrag des Generalanwaltes RuizJarabo Colomer v. 12.10.2009 im Verfahren Nutral SpA ./. Kommission der Europäischen Gemeinschaften, Rs. C-476/93 P, Slg. 1995, I-04125, 4131, Rn. 17; vgl. a. zum Recht auf Verteidigung EuGH, Urt. v. 16.9.2009, Limburgse Vinyl Maatschappij u.a.,in den verbundenen Rechtssachen T-305 bis 307/94, pp, Slg. 1999, II-931, Rn. 246. Zu den Verfahrensrechten allgemein: Jokisch Gemeinschaftsrecht und Strafverfahren, 2000, S. $71 \mathrm{ff}$.

38 Gless/Zeitler European Law Journal 2001, 219.

${ }^{39} \mathrm{Vgl}$. EuGH, Urt. v. 3.9.2008, Kadi ./. Rat und Kommission, Rs. C-402/05 und C-415/05; zuvor Urt. v. 21.9.2005, Kadi ./. Rat und Kommission, T-315/01; Urt. v. 21.9. 2005, Yusuf und Al Barakaat International Foundation ./. Rat und Kommission, Rs. T-366/01.

${ }^{40}$ Klip European Criminal Law, Cambridge 2012; Gleß ZStW 116 (2004), 353.

41 Gless StV 2013, 317.

${ }^{42} \operatorname{SEK}(2001) 380,3$.

${ }^{43} \mathrm{KOM}(2005) 172$ endg. 
nicht nur in einem ersten Gesetzgebungsentwurf, sondern während des ganzen Rechtsetzungsverfahrens bedürfe. ${ }^{44}$ Gleichwohl fehlt der generellen Verpflichtung ein adäquater Fokus auf die spezifischen Bedürfnisse des Strafverfahrens ebenso wie ein striktes follow-up. ${ }^{45}$

\section{Etablierung von Beschuldigtenrechten durch die Richtlinie 2012/13}

Eine strikte Rechtsregelung könnte man nun in der Richtlinie 2012/13 sehen, die einen wichtigen Aspekt einer effektiven Verteidigung in der gesamten EU sichern soll. ${ }^{46}$ Die Forderung nach einer Stärkung der Verteidigung gehört auch zu den Kernanliegen des von Bernd Schünemann initiierten europapolitischen Projektes. ${ }^{47}$ Doch um das Ergebnis vorweg zu nehmen: Der Jubilar würde die europäische Vorgabe in der Richtlinie 2012/13 und deren Umsetzung in Deutschland kaum uneingeschränkt begrüßen, sondern wohl eher in die von verschiedenen Seiten geäußerte Kritik einstimmen. ${ }^{48}$ Diese richtet sich, jenseits der Einwände gegen das in der Richtlinie 2010/64/EU ebenfalls verankerte Recht auf einen Dolmetscher, ${ }^{49}$ vor allem auf die verpasste Chance (a) durch die Richtlinie eine europäische Vision von europaweiten Beschuldigtenrechten aufzubauen und (b) die Geltung von Menschen- und Grundrechten einheitlich zu verankern.

a) Europäische Kompensation für die Strafverteidigung angesichts europaweiter Strafverfolgung

Bernd Schünemann forderte von Anfang an nicht nur eine Minimallösung, etwa eine erweiterte Belehrungspflicht, sondern eine umfassende Antwort auf das Kompensationsproblem, also Garantien für eine wirksame Verteidigung des Beschuldigten in einem europäisierten oder gar von einem Europäischen Staatsanwalt geführten Ermittlungsverfahren. ${ }^{50}$

Nun ist der Europäische Staatsanwalt zwar noch nicht eingeführt, gleichwohl darf man mit Blick auf den von der EU-Kommission bereits vorgelegten Vorschlag ${ }^{51}$ und angesichts des kontinuierlichen Ausbaus der europäischen

${ }^{44}$ Mitteilung der Kommission: Strategie zur wirksamen Umsetzung der Charta der Grundrechte durch die Europäische Union, KOM (2010) 573 endg.

45 Vgl. Schünemann GA 2002, 193; Gless StV 2013, 317.

${ }^{46} \operatorname{Nestler}$ (Fn. 10), S. 167.

${ }^{47}$ Nestler (Fn. 10), S. $166 \mathrm{ff}$.

${ }^{48}$ Vgl. dazu: Esser FS Wolter, 2013, S. 1329, $1330 \mathrm{ff}$.

49 Ausf. dazu Yalcin ZRP 2013, 104; Wawzyniak Plenarprotokoll 17/240, Stenographischer Bericht der 240. Sitzung des Deutschen Bundestags vom 16. Mai 2013, 30428.

50 Schünemann $\mathrm{StV}$ 2006, 361, $367 \mathrm{f}$.

51 Vgl. zum Vorschlag der EU-Kommission sowie zu den Vorschlägen der Expertenkommission: Ligeti Toward a prosecutor for the European Union, Oxford 2013; Grünewald HRRS 2013, 508, 509. 
Strafverfolgung dem EU-Gesetzgeber durchaus vorwerfen, dass die EU-Richtlinie in der Substanz auf bloße Deckungsgleichheit mit den Mindestanforderungen der EMRK ${ }^{52}$ und damit auf die Festlegung europäischer Minimalrechte auf staatlicher Ebene zielt. ${ }^{53}$ Das erscheint rechtspolitisch gefährlich und von einer Kurzsichtigkeit geprägt, vor der Bernd Schünemann gewarnt hat.

Zum ersten wird dem Anliegen einer adäquaten strukturellen Stärkung der Verteidigung als Ausgleich zum flächendeckenden Ausbau einer europäischen Strafverfolgung nicht Rechnung getragen. ${ }^{54}$ Das ist vor allem deshalb misslich, weil die Zusammenarbeit zwischen den europäischen Staaten auf dem Gebiet der Strafverfolgung gerade in jüngerer Zeit mit dem Hinweis vorangetrieben wird, dass gleichzeitig die Rechte der Verteidigung durch neue Richtlinien verstärkt würden. ${ }^{55}$ Es ist jedoch keine zentrale Institution zur Sicherung einer adäquaten europaweiten Strafverfolgung in Sicht, wie sie etwa als Eurodefensor oder als European Criminal Law Ombudsman, ${ }^{56}$ von dem Kreis um Bernd Schünemann gefordert wurden. ${ }^{57}$ Ohne diese strukturellen Maßnahmen vergrößert sich jedoch die Ungleichheit der Kräfte zwischen vernetzten Strafverfolgungsbehörden und nur im Einzelfall tätigen Strafverteidigern. Die Kritik an diesem Versäumnis trifft aber nicht nur den europäischen Gesetzgeber, sondern auch die Umsetzung der Richtlinie in Deutschland. Die EU-Vorgaben sehen explizit die Möglichkeit eines weitergehenden Schutzes vor. ${ }^{58}$ Gleichwohl beschränkt sich auch der deutsche Gesetzgeber auf eine gesetzliche Verankerung des Mindeststandards und wird damit dem eigenen Anspruch nicht gerecht, Vorbild in Europa für die Wahrung von Grund- und Menschenrechten im Strafprozess zu sein. ${ }^{59}$

\section{b) Grundrechts- und Menschenrechtsbindung europäischer Strafrechtspflege}

Darüber hinaus wirft die EU-Rechtlinie und ihre Umsetzung ein neues Schlaglicht auf das grundsätzliche Problem des Grundrechts- und Menschenrechtsschutzes auf europäischer Ebene: das undurchsichtige Nebeneinander

52 Löwe/Rosenberg/Esser, 26. Aufl. 2012, Art. 6 EMRK Rn. 566.

53 Vgl. RL 2012/13/EU, Präambel (7) ff., auch wenn der Rechtsakt nach der Intention einen „proaktiven Ansatz“ verfolgen und den von LR/Esser (Fn. 52), Art. 6 EMRK Rn. 566 dargestellten EMRK-rechtlichen status quo festschreiben wollte: vgl. a. Nestler (Fn. 10), 167.

${ }^{54} \mathrm{Zu}$ dieser Problematik etwa: Schünemann StV 2006, 361, 362, 367; Gless StV 2013, 317; Nestler (Fn. 10), 168.

55 Vgl. RL 2012/13/EU, Präambel (10).

56 Dazu ausf. Mitchell in:Schünemann (Hrsg.), Gesamtkonzept für die europäische Strafrechtspflege - A Programme for European Criminal Justice, 2006, S. 191, 193 ff.

57 Mitchell (Fn. 56), sowie Nestler (Fn. 10)., S. 193 ff. und 172 ff.; Schünemann StV 2006, 361, $367 \mathrm{f}$.

58 RL 2012/13/EU, Präambel (40).

59 Vgl. etwa Wawzyniak (Fn. 49), 30428. 
unterschiedlich formulierter Rechte, die in der Substanz alle auf das Gleiche zielen (sollten).

Die Richtlinie 2012/13 selbst beschreibt ihre grundrechtliche Verankerung im Wesentlichen in den Erwägungsgründen 5-8. ${ }^{60}$ Die Ausführungen verweisen auf die unterschiedlichen Rechte aus Art. 48 der Charta der Grundrechte der Europäischen Union (EU-Charta) und Art. 6 EMRK ohne große Differenzierung, was mehr verwirrt als klärt. Denn während Art. 48 Abs. 2 EUCharta dem Wortlaut nach nur "die Achtung der Verteidigungsrechte“ gewährleistet, wie sie in den mitgliedstaatlichen Rechtsordnungen bereits bestehen, gewährt Art. 6 EMRK bekanntlich gerade ein von nationalen Gesetzen unabhängiges Recht auf effektive Verteidigung, das durch verschiedene Teilrechte des Europäischen Gerichtshofs für Menschenrechte (EGMR) etabliert wurde. Den Konflikt zwischen beiden Verweisen löst die Richtlinie nicht auf, sondern erklärt in Erwägungsgrund 14 lediglich: „Diese Richtlinie baut auf den in der Charta verankerten Rechten auf, insbesondere auf den Artikeln 6, 47 und 48 der Charta und legt dabei die Artikel 5 und 6 EMRK in der Auslegung durch den Europäischen Gerichtshof für Menschenrechte zugrunde.“ Im Rechtstext selbst fixiert Art. 10 Richtlinie 2012/13 ein „Regressionsverbot“: „Keine Bestimmung dieser Richtlinie ist so auszulegen, dass dadurch die Rechte oder Verfahrensgarantien nach Maßgabe der Charta, der EMRK, anderer einschlägiger Bestimmungen des Völkerrechts, oder der Rechtsvorschriften der Mitgliedstaaten, die ein höheres Schutzniveau vorsehen, beschränkt oder beeinträchtigt werden." Was das im Klartext bedeuten soll, bleibt unklar. Denn es sind zu viele Unbekannte im Spiel - im Verhältnis zwischen den Belehrungspflichten, die sich einerseits (bereits) aus der EMRK und andererseits aus den - durch EU-Vorgaben veranlassten - neuen nationalen Verfahrensvorschriften ergeben.

Das Spannungsfeld zwischen nationalen Vorgaben, EMRK- Pflichten und (über die Grundrechtecharta bei Umsetzung von EU-Vorgaben ins nationale Recht transponierter) EU-Bindung hat exemplarisch das „Fransson“-Urteil des EuGH aufgezeigt: ${ }^{61}$ Nach Aussage des EuGH bestimmt das Unionsrecht zwar weder das Verhältnis zwischen der EMRK und den Rechtsordnungen der Mitgliedstaaten, noch die Konsequenzen, die ein nationales Gericht aus einem Widerspruch zwischen den durch die EMRK gewährleisteten Rechten und einer nationalen Rechtsvorschrift zu ziehen hat. Der EuGH verlangt jedoch, dass die Gerichte der Mitgliedstaaten die durch die Charta verbürgten Rechte auch innerstaatlich durchsetzen. Ein nationales Gericht, das Unionsrecht anwendet, sei gehalten, „für die volle Wirksamkeit dieser Normen Sorge zu tragen, indem es erforderlichenfalls jede - auch spätere - entgegen-

60 RL 2012/13/EU.

61 EuGH, Urt. v. 26.2.2013, Åklagare ./. Hans Åkerberg Fransson, Rs. C-617/10. 
stehende Bestimmung des nationalen Rechts aus eigener Entscheidungsbefugnis unangewandt lässt". ${ }^{62}$ Die von Art. 51 Abs. 1 Grundrechtecharta vorausgesetzte „Durchführung des Rechts der Union“63 ist bei jeder richterlichen oder exekutiven Anwendung von nationalen Rechtsvorschriften gegeben, die eine EU-Richtlinie oder einen EU-Rahmenbeschluss umsetzt. ${ }^{64}$ Dies gilt also auch für $\$ 136$ Abs. 1 Satz 2 StPO n.F.

\section{Der EU-Gesetzgeber in der Konsequenz - doch (k)ein guter Geist?}

Gleichwohl bleibt festzuhalten: $\ 136$ Abs. 1 Satz 2 StPO ist eine Verbesserung der Rechtsstellung des einzelnen, initiiert durch die „Brüssler Strafrechtspflege“. Hat letztere dadurch nun eine Richtungskorrektur vollzogen, aufgrund derer sie für sich in Anspruch nehmen darf, sich vom Gespenst jedenfalls punktuell - in einen guten Geist verwandelt zu haben?

\section{In der Praxis dentscher Strafjustiz}

Auf den ersten Blick scheint die Antwort positiv. Das Gesetz zur Stärkung der Verfahrensrechte von Beschuldigten im Strafverfahren bewirkt mit der Ergänzung des $\$ 136$ StPO zunächst das, was es auch verspricht: eine Besserstellung beschuldigter Personen durch einen Anspruch auf ausführlichere Belehrung über ihre Rechte.

Doch dieser Anspruch auf die Belehrung über Pflichtverteidigung taugt natürlich nur dann etwas, wenn dem Beschuldigten am Ende auch ein real hilfreicher Strafverteidiger zur Seite steht. Voraussetzung dafür ist unter anderem, dass etwa die Qualität der Strafverteidigung durch flankierende Maßnahmen zur Sicherung von entsprechender Qualifikation und Entlohnung von Pflichtverteidigern gewährleistet ist. ${ }^{65}$ Entsprechende Regelungen fehlen aber in der Richtlinie. Dass die frisch verankerte Belehrungspflicht

${ }^{62}$ EuGH, Urt. v. 26.2.2013, Åklagare ./. Hans Åkerberg Fransson, Rs. C-617/10, Rn. 44, 45 mit weiteren Verweisen; kritisch zur EuGH-Rechtsprechung etwa Rabe NJW 2013, 1407.

${ }^{63}$ Mit Verweis auf die Rechtsprechung EuGH, Urt. v. 18.6.1991, ERT, Rs. C-260/89.

${ }^{64}$ Wegner HRRS 2013, 126, 127. Zur Diskussion vor der „Fransson“-Entscheidung EuGH, Urt. v. 12.12.1996, Strafverfahren gegen X, Rs. C-74/95, Rn. 25; Borowsky/Ehlers in: Ehlers (Hrsg.), Europäische Grundrechte und Grundfreiheiten, 2009, $\mathbb{1} 14$ V 2 Rn. 51; strittig ist, ob die Grundrechtecharta auch dann anwendbar ist, wenn eine nationale Norm zwar der Umsetzung einer Richtlinie dient, sich dabei aber noch im nationalen Umsetzungsspielraum bewegt, dazu: Kingreen in: Callies/Ruffert (Hrsg.), EUV/AEUV, 4. Aufl. 2011, Art. 51 GRCh Rn. 10 ff. m.w.N.

${ }^{65} \mathrm{Vgl}$. Nestler (Fn. 10), S. 167. 
neue praktische Schwierigkeiten in den einzelnen Rechtsordnungen schafft, ist wohl kaum zu vermeiden. So dürfte etwa in Deutschland die Notwendigkeit einer Verteidigerbestellung nach $\ 140$ Absatz $2 \mathrm{StPO}^{66}$ vor der ersten Vernehmung des Beschuldigten in verschiedenen Fallkonstellationen nicht genau feststehen, ${ }^{67}$ insbesondere noch im Ermittlungsverfahren ( $\ 141$ Absatz 3 StPO). Fraglich ist, wie sich insofern die vom deutschen Gesetzgeber gewählte, doch recht pauschale Formulierung in $\$ 136$ Abs. 1 Satz 2 StPO mit der Verpflichtung zur effizienten Umsetzung der EU-Richtlinie vereinbaren lässt.

Darüber hinaus bleiben ungelöste Fragen, etwa die nach den Konsequenzen eines möglichen Verstoßes gegen die Belehrung im jeweiligen Strafverfahren nicht europaweit einheitlich geregelt sind. Das beginnt mit der Frage, was Pflichtverteidigung in concreto bedeutet und geht bis zu der Frage, ob ein Verstoß gegen die Belehrungspflicht überhaupt Folgen in dem jeweiligen Strafverfahren nach sich zieht. Aus deutscher Sicht sollte, wie bereits erläutert, ein Beweisverwertungsverbot eingreifen, während sich in anderen Staaten die Frage gar nicht oder nicht in gleicher Weise stellt. ${ }^{68}$

\section{Im europäischen Kräftefeld}

Welche praktischen Konsequenzen sich aus diesem europäischen Kräftefeld für die Belehrung über eine unentgeltliche Verteidigung in concreto ergeben, bleibt offen. Die Grundrechtecharta selbst enthält eben keinen ausdrücklichen Hinweis auf Belehrungsrechte. Gleichwohl wird angenommen, dass sie die Vorgaben der EMRK und der Rechtsprechung des EGMR zur „Achtung der Verteidigungsrechte" (Art. 48 Abs. 2 EU-Charta) als Verpflichtung zur Gewährleistung einer effektiven Verteidigung übernimmt und für die Mitgliedstaaten im EU-Kontext unmittelbar verbindlich macht. ${ }^{69}$

Damit bleibt das Recht auf Verteidigung nach Art. 6 Abs. 3 lit. c EMRK die bestimmende europäische Kraft für eine europäische Strafverteidigung. Der EGMR hat die Fahrrinne durchaus früh vorgespurt und in seiner Rechtsprechung hergeleitet, dass das Recht auf effektive Verteidigung in bestimmten Fällen, z.B. bei besonderer Schutzwürdigkeit des Beschuldigten, impli-

${ }^{66} \mathrm{Vgl}$. zu der mit der kasuistischen Ausdifferenzierung verbundenen Unübersichtlichkeit, LR/Lüderssen/Jahn, 26. Aufl. 2007, $\$ 140$ Rn. 50.

${ }^{67} \mathrm{Vgl}$. a. Neubaus StV 2010, 45.

${ }^{68}$ Höpfel/Huber Beweisverbote in Ländern der EU und vergleichbaren Rechtsordnungen, 1999.

${ }^{69}$ In diesem Sinne Callewaert EuGRZ 2003, 198, 200; Winkler EuGRZ 2001, 18, 23 ff.; Borowsky in: Meyer (Hrsg.), Charta der Grundrechte der Europäischen Union, 3. Aufl. 2010, Art. 52 Rn. 34, unter Hinweis auf Art. 52 Abs. 3 EU-Charta; siehe auch Wegner HRRS 2013, 126, 130. 
ziert, dass der Beschuldigte über sein Recht auf einen unentgeltlichen Verteidiger belehrt wird. ${ }^{70} \mathrm{Da}$ die staatlichen Organe dafür sorgen müssten, dass der Beschuldigte das Recht auf Verteidigung effektiv wahrnehmen kann, verlangt die EMRK eine adäquate Information über die Verteidigungsrechte, was die Belehrung über das Recht auf unentgeltlichen Rechtsbeistand soweit ein solcher zur Verfügung steht - einschließt. ${ }^{71} \mathrm{Ob}$ Deutschland mit dem Gesetz zur Stärkung der Verfahrensrechte von Beschuldigten seiner vielschichtigen europäischen Pflicht, zusammengesetzt aus den Verpflichtungen der EMRK und deren Transposition über die EU-Grundrechtecharta in das nationale Recht, adäquat nachgekommen ist, bleibt abzuwarten. Möglicherweise weist am Ende doch die vom EGMR zur Souveränitätswahrung der Vertragsstaaten bereits seit langem zugrunde gelegte sog. margin of appreciation-doctrine einen Ausweg: ${ }^{72}$ Danach genießen die Vertragsstaaten einen gewissen Ermessensspielraum bei der Umsetzung von EMRK-Rechten. ${ }^{73}$ Der EGMR untersucht, ob es unter den Vertragsstaaten einen gemeinsamen Standard gibt, und wendet dann die Regel an: Je geringer ausgebildet der gemeinsame Standard ist, desto größeren Ermessensspielraum haben die Vertragsstaaten. ${ }^{74}$ Das hat allerdings nicht die - auf EU-Ebene gewünschte Harmonisierung zur Folge, ${ }^{75}$ und führt so wieder zurück zur offenen Frage der konkreten Menschen- und Grundrechtsgeltung.

70 Vgl. EGMR, Urt. v. 11.12.2008, Panovits ./. Zypern, Appl. no. 4268/04, Rn. 68; Urt. v. 9.6.1998, Twalib ./. Griechenland, Appl. no. 24294/94, Rn. 55; vgl. auch Woblers FS Rudolphi, 2004, S. 713, 727 ff.; LR/Esser (Fn. 52), Art. 6 EMRK Rn. 755 unter Verweis auf Art. 14 Abs. 3 lit. d IPBPR.

${ }_{71}$ Vgl. EGMR, Urt. v. 11.12.2008, Panovits ./. Zypern, Appl. no. 4268/04, Rn. 68: “The Court considers that given the vulnerability of an accused minor and the imbalance of power to which he is subjected by the very nature of criminal proceedings, a waiver by him or on his behalf of an important right under Article 6 can only be accepted where it is expressed in an unequivocal manner after the authorities have taken all reasonable steps to ensure that he or she is fully aware of his rights of defence and can appreciate, as far as possible, the consequence of his conduct"; ebenso EGMR, Urt. v. 8.4.2010, Sinichkin ./. Russland, Appl. no. 20508/03, Rn. 35 und 48. Aus deutscher Sicht: LR/Lüderssen/Jahn (Fn. 18), $\$ 137$ Rn. 66b.

72 Peters/Altwicker Europäische Menschenrechtskonvention: Mit rechtsvergleichenden Bezügen zum deutschen Grundgesetz, 2012; Gless/Martin Bergen Journal of Criminal Law \& Criminal Justice 1 (2013), 36.

${ }^{73}$ LR/Esser (Fn. 52), Art. 6 EMRK Rn. 179.

${ }^{74}$ EGMR, Urt. v. 26.4.1979, Sunday Times ./. das Vereinte Königreich, Appl. no. 6538/74, Rn. 59.

75 Vgl. dazu etwa EGMR, Urt. v. 22.10. 2009, Raykov ./. Bulgarien, Appl. no. 35185/03, Rn. 65; Urt. v. 27.4.2006, Sannino ./. Italien, Appl. no. 30961/03, Rn. 48; Urt. v. 13.1.2009, Rybacki ./. Polen, Appl. no. 52479/99, Rn. 54; Urt. v. 12.2.2009, Samokhvalov ./. Russland, Appl. no. 3891/03, Rn. 56; Urt. v. 31.3.2009, Plonka ./. Polen, Appl. no. 20310/02, Rn. 33; Urt. v. 8.12.2009, Caka ./. Albanien, Appl. no. 44023/02, Rn. 85; Urt. v. 2.11.2010, Sakhnovski ./. Russland, Appl. no. 21272/03, Rn. 95. 


\section{Fazit}

Und so spukt die „Brüssler Strafrechtspflege“ weiter durch Europa. Allerdings tritt sie uns heute in anderer Gestalt gegenüber als es das Gespenst getan hat, dem wir mit Bernd Schünemann vor über einer Dekade begegnet sind. Die Erscheinung ist vielschichtiger geworden. Während zu Beginn der europäischen Zusammenarbeit klar die Anliegen der Strafverfolgung alleine das Bild bestimmt haben, treten nun langsam Individualinteressen - Beschuldigten- und Opferrechte - mit in den Vordergrund. Noch lange aber ist das Gebilde kein ausgewogenes Ganzes. Deshalb wird weiter um Legitimation und Grenzen gemeinsamer Strafverfolgung in Europa gerungen werden. Auch $₫ 136$ Abs. 1 S. 2 StPO könnte zu einem Schauplatz des Ringens Brüsseler und Berliner (Kriminal-)Politik um die Definitionsmacht über Justizund Grundrechte im Strafverfahren werden. Denn nicht nur die nationalen Gerichte werden die neu etablierte Pflicht zur Belehrung über den unentgeltlichen Rechtsbeistand konkretisieren müssen, auch die Jurisdiktion des EuGH ist im Grundsatz geöffnet, weil die Gesetzesänderung auf der Umsetzung einer EU-Richtlinie beruht und damit eine starke Verknüpfung der nationalen Normen mit dem Recht der Union ${ }^{76}$ besteht.

Bernd Schünemann wird sich in dieser Diskussion sicherlich weiter mit Gewinn für alle zu Wort melden. Für die Präsentation seiner Arbeiten am Gesamtkonzept für eine europäische Strafrechtspflege hat er Griechenland als Wiege der Demokratie gewähltt. ${ }^{77}$ Griechenland hat Europa daneben noch vieles zu bieten, unter anderem den gemeinsamen - und insbesondere bei Bernd Schünemann beliebten - Sagenschatz, der auch berichtet, dass die Herausforderung Europa selbst die Götter des Olymps an und über ihre Grenzen gebracht hat. ${ }^{78}$ Wer mit Europa geht, muss sich öffnen. Die Zeit ist vorbei, in der man in Deutschland glaubte, der europäische Geist könne eigentlich nichts zur Verbesserung der deutschen Strafrechtspflege beitragen. ${ }^{79}$ Europa kann vom guten Geist Deutschlands und Deutschland kann auch vom guten Geist Europas profitieren.

${ }^{76}$ EuGH, Urt. v. 26.2.2013, Åklagare ./. Hans Åkerberg Fransson, Rs. C-617/10, Rn. 16.

77 Schünemann (Fn. 9), S. VIII.

${ }_{78}$ Gless StV 2010, 400.

79 Vgl. etwa noch Roxin Strafverfahrensrecht, 24. Aufl. 1995, $\mathbb{3}$ Rn. 7, der die praktische Bedeutung der Garantien der EMRK für das deutsche Strafprozessrecht als relativ gering einschätzte, siehe jetzt aber Roxin/Scbünemann Strafverfahrensrecht, 28. Aufl. 2014, $\int 3 \mathrm{Rn} .14 \mathrm{ff}$. 